\title{
KAM 环面附近粘性解的正则性
}

\author{
梁振国, 严军
}

复旦大学数学科学学院、教育部非线性数学模型重点实验室, 上海 200433

E-mail: zgliang@fudan.edu.cn, yanjun@fudan.edu.cn

收稿日期: 2007-4-10; 接受日期: 2007-10-15

国家自然科学基金 (批准号: 10701027，10601013) 和上海市科研基金 (批准号: 06JC14005) 资助项目

摘要 给出了 $u^{\varepsilon}(x, P)$ 和 $D u^{\varepsilon}(x, P)$ 关于 $P$ 的一个弱的正则性定理, 这里 $u^{\varepsilon}(x, P)$ 是方 程 (1.1) 的粘性解.

关键词 粘性解 KAM 环面 Hamilton-Jacobi 方程

$\operatorname{MSC}(2000)$ 主题分类 $37 \mathrm{~J} 40,49 \mathrm{~L} 25,35 \mathrm{C} 20$

\section{1 内容介绍及主要结果}

众所周知, 粘性解的正则性对于进一步的了解 Hamilton 动力系统是非常重要的. 本文将 研究近可积的 Hamilton 系统, 其 Hamilton 量形为

$$
H_{\varepsilon}(p, x)=H_{0}(p)+\varepsilon H_{1}(p, x),
$$

这里 $H_{0}$ 和 $H_{1}$ 光滑, $H_{0}$ 严格凸, $H_{1}(p, x)$ 的 0 阶导数及直到任意阶导数均有界, 并且其关于 $x$ 变量 $\mathbb{Z}^{n}$ 周期. 我们将研究 $u^{\varepsilon}(x, P)$ 及 $D u^{\varepsilon}(x, P)$ 关于 $P$ 的依赖性, 这里 $u^{\varepsilon}(x, P)$ 是方程

$$
H_{\varepsilon}\left(P+D_{x} u^{\varepsilon}, x\right)=\bar{H}_{\varepsilon}(P)
$$

的粘性解, 并且 $P$ 限制在 $P_{\varepsilon}$ 的邻域中 (对 $P_{\varepsilon}$ 的解释, 见文献 [1]).

为叙述方便, 我们将先给定一个参考值 $P=P_{0}$. 假设旋转向量 $\omega_{0}=D_{P} \bar{H}_{0}\left(P_{0}\right)$ 满足 Diophantine 条件

$$
\left|\left\langle k, \omega_{0}\right\rangle\right| \geqslant \frac{C}{|k|^{\tau}},
$$

这里 $C$ 是正常数, $\tau>n-1$. 在文献 [1] 中, Gomes 证明了在小摄动下 Diophantine 不变环面 和 Aubry-Mather 集均是稳定的. 除此之外, 他还得到了下面两个估计: 对任意的 $M>0$, 存 在着 $N(M)$ 满足估计

$$
\begin{aligned}
& \sup _{x}\left|u^{\varepsilon}\left(x, P_{\varepsilon}\right)-\tilde{u}_{N}^{\varepsilon}\left(x, P_{\varepsilon}\right)\right| \leqslant \cdot \varepsilon^{M}, \\
& \underset{x}{\operatorname{esssup}}\left|D_{x} u^{\varepsilon}\left(x, P_{\varepsilon}\right)-D_{x} \tilde{u}_{N}^{\varepsilon}\left(x, P_{\varepsilon}\right)\right| \leqslant \cdot \varepsilon^{\frac{M}{2}} .
\end{aligned}
$$


上面的两个估计只对 $P_{\varepsilon}$ 成立. 本文的第 1 个结果把上述估计推广到 $P_{\varepsilon}$ 的一个邻域中 去. 具体而言, 在定理 1 和 2 中, 我们证明了: 对于任何 $M>0$, 均存在 $N(M)$, 使得对于任 何满足 $\left|P-P_{\varepsilon}\right| \leqslant \cdot \varepsilon^{\alpha}\left(\alpha \geqslant \frac{N}{4}\right)$ 的 $P$, 上面估计 (1.3) 均成立. 基于上面结论, 我们得到了关 于 $u^{\varepsilon}(x, P)$ 和 $D u^{\varepsilon}(x, P)$ 在 $P_{\varepsilon}$ 的一个小邻域的 $P$ 的弱正则性结果. 这是我们的定理 3 和 4. 我们证明了对于满足 $\left|P-P_{\varepsilon}\right| \leqslant \cdot \varepsilon^{8}$ 的任何 $P$, 均存在一个 $k_{0}$, 若 $\cdot \varepsilon^{2^{k_{0}+4}} \leqslant\left|P-P_{\varepsilon}\right| \leqslant$ $\cdot \varepsilon^{2^{k_{0}+3}}\left(k_{0}=0,1, \ldots\right)$, 则有估计式

$$
\begin{aligned}
& \left\|u^{\varepsilon}(x, P)-u^{\varepsilon}\left(x, P_{\varepsilon}\right)\right\| \leqslant \cdot\left|P-P_{\varepsilon}\right|^{\frac{1}{2^{k}{ }^{+4} \tau}}, \\
& \underset{x}{\operatorname{esssup}}\left|D_{x} u^{\varepsilon}(x, P)-D_{x} u^{\varepsilon}\left(x, P_{\varepsilon}\right)\right| \leqslant \cdot\left|P-P_{\varepsilon}\right|^{\frac{1}{2^{k_{0}+5} \tau}} .
\end{aligned}
$$

成立.

这里的指数 $\cdot \frac{1}{\tau}$ 并不奇怪. 回忆 Mather 关于 Peierls's Barrier (参见文献 [2]) 的工作. Mather 证明了若 $\omega$ 满足 $\tau$ 阶的 Diophantine 条件, 则 $\omega \rightarrow P_{\omega}(\xi)$ 满足 $\left|P_{\omega}(\xi)-P_{\rho}(\xi)\right| \leqslant$ const $\mid \omega-\rho^{*} \frac{1}{2 \tau}$, 这里要求 $\left|\omega-\rho^{*}\right| \leqslant 1$. 注意到这里的 Peierls's Barrier 函数能由粘性解表出, 从而上式也能表达成粘性解的形式.

简单回顾一下粘性解的相关文献. 文献 [3, 4] 中, 对 Hamilton-Jacobi 方程的粘性解理论 有一个比较全面的介绍. 关于这一理论后续的发展, 参见文献 [5-9].

\section{2 经典的摄动方法及粘性解的一致逼近}

为完整起见, 我们将重复文献 [1] 中的方法. 下面的做法目的是寻找方程

$$
H_{\varepsilon}\left(P+D_{x} u^{\varepsilon}, x\right)=\bar{H}_{\varepsilon}(P)
$$

的一个近似解, 这里 $P=P_{0}+O(\varepsilon)$. 众所周知, KAM 的直接方法通过迭代的方法来构造方 程 $(2.1)$ 形如

$$
u^{\varepsilon}=u_{0}+\varepsilon v_{1}+\varepsilon^{2} v_{2}+\cdots
$$

的解. 主要的技术困难在于证明上面展开式的收玫性. 我们这里只需求解前面有限项, 进而用 之去逼近 $u^{\varepsilon}$.

明显的是当 $\varepsilon=0, \bar{H}_{0}(P)=H_{0}(P)$, 此时, 解 $u^{0}$ 是常数. 不妨取 $u^{0} \equiv 0$. 当 $\varepsilon>0$ 时, 形 式上有 $u^{\varepsilon}=O(\varepsilon)$. 我们采用近似解 $\tilde{u}_{N}^{\varepsilon}$ 逼近粘性解 $u^{\varepsilon}$, 这里

$$
\begin{aligned}
\tilde{u}_{N}^{\varepsilon}= & \varepsilon v_{1}\left(x, P_{0}\right)+\varepsilon\left(P-P_{0}\right) D_{P} v_{1}\left(x, P_{0}\right)+\varepsilon^{2} v_{2}\left(x, P_{0}\right) \\
& +\frac{1}{2} \varepsilon\left(P-P_{0}\right)^{2} D_{P P}^{2} v_{1}\left(x, P_{0}\right)+\varepsilon^{2}\left(P-P_{0}\right) D_{P} v_{2}\left(x, P_{0}\right) \\
& +\varepsilon^{3} v_{3}\left(x, P_{0}\right)+\cdots .
\end{aligned}
$$

这里相当于把 $u^{\varepsilon}$ 的展开式展开到 $N-1$ 阶, 形式上说 $u^{\varepsilon}-\tilde{u}_{N}^{\varepsilon}=O\left(\varepsilon^{N}\right)$. 上面提到的函数 $v_{i}$ 和 $D_{P^{k}}^{k} v_{i}$ 满足 transport 方程

$$
D_{p} H_{0}\left(P_{0}\right) D_{x} w=f(\cdots),
$$

并且能通过迭代的方法求出. $v_{i}(x, P)$ 以及 $D_{P k}^{k} v_{i}$ 能通过求解相应的 transport 方程得出. 除 此之外, 相应的其他常数也可通过积分协调性条件得出, (参见文献 [1]).

若 $H$ 充分光滑并且 $\omega_{0}$ 非共振, 则上述方程有光滑解, 并且在相差一个常数的意义下是 唯一的. 直接验证可知

$$
H_{\varepsilon}\left(P+D_{x} \tilde{u}_{N}^{\varepsilon}, x\right)=\tilde{H}_{\varepsilon}^{N}(P)+O\left(\varepsilon^{N}+\left|P-P_{0}\right|^{N}\right)
$$


成立, 这里

$$
\begin{aligned}
\tilde{H}_{\varepsilon}^{N}(P)= & \bar{H}_{0}\left(P_{0}\right)+\varepsilon \bar{H}_{1}\left(P_{0}\right)+D_{P} \bar{H}_{0}\left(P_{0}\right)\left(P-P_{0}\right)+\cdots \\
& +\frac{1}{(N-1) !} \frac{\partial^{N-1} \bar{H}_{0}}{\partial P^{N-1}}\left(P_{0}\right)\left(P-P_{0}\right)^{N-1}+\cdots+\varepsilon^{N-1} \bar{H}_{N-1}\left(P_{0}\right),
\end{aligned}
$$

且

$$
\begin{aligned}
\tilde{u}_{N}^{\varepsilon}= & \varepsilon v_{1}\left(x, P_{0}\right)+\left(\varepsilon \frac{\partial v_{1}}{\partial P}\left(x, P_{0}\right)\left(P-P_{0}\right)+\varepsilon^{2} v_{2}\left(x, P_{0}\right)\right)+\cdots \\
& +\left(\varepsilon \frac{1}{(N-2) !} \frac{\partial^{N-2} v_{1}}{\partial P^{N-2}}\left(x, P_{0}\right)\left(P-P_{0}\right)^{N-2}+\cdots+\varepsilon^{N-1} v_{N-1}\left(x, P_{0}\right)\right) .
\end{aligned}
$$

注 2.1 事实上, 对于 $\left|P-P_{0}\right| \leqslant \cdot \varepsilon+\cdot \varepsilon^{\alpha}(\alpha>1)$, 有

$$
\left|H_{\varepsilon}\left(P+D_{x} \tilde{u}_{N}^{\varepsilon}, x\right)-\tilde{H}_{\varepsilon}^{N}(P)\right| \leqslant \cdot \varepsilon^{N} .
$$

下面的推论同样来自文献 [1] 的推论 1 和 2 .

引理 2.1 对于任何 $P=P_{0}+O(\varepsilon)$ (即 $\left|P-P_{0}\right| \leqslant c \varepsilon$, 这里的常数 $c$ 不依赖于 $P$ 的选择, 下同), 存在 $x_{0}$ 满足

$$
D_{x} u^{\varepsilon}\left(x_{0}, P\right)=D_{x} \tilde{u}_{N}^{\varepsilon}\left(x_{0}, P\right)
$$

这里 $x_{0}$ 依赖于 $P$.

引理 2.2

$$
\bar{H}_{\varepsilon}(P)=\tilde{H}_{\varepsilon}^{N}(P)+O\left(\varepsilon^{N}+\left|P-P_{0}\right|^{N}\right) .
$$

注 2.2 从上述引理 2.1 和 2.2 的证明及注 2.1 , 我们有

$$
\left|\bar{H}_{\varepsilon}(P)-\tilde{H}_{\varepsilon}^{N}(P)\right| \leqslant \cdot \varepsilon^{N} .
$$

记 $\omega(P)=D_{P} \tilde{H}_{\varepsilon}^{N}(P)$. 从文献 [1], 知存在 $P_{\varepsilon}$, 使得 $\omega\left(P_{\varepsilon}\right)=\omega_{0}$ 成立. 若 $P$ 充分接近 $P_{\varepsilon}$, 我们将有下述引理, 其证明可在附录中找到:

引理 2.3 若 $\left|P-P_{\varepsilon}\right| \leqslant \cdot \varepsilon^{\alpha}$, 则 $\left|\omega(P)-\omega_{0}\right| \leqslant \cdot \varepsilon^{\alpha}$.

本文的第 1 个定理如下:

定理 2.1 假设旋转向量 $\omega_{0}$ 满足 Diophantine 条件 (1.2). 对于任意 $M>0$, 存在 $N(M)$ 和 $B\left(P_{\varepsilon}, \cdot \varepsilon^{\alpha}\right)$ (即 $\left.\left|P-P_{\varepsilon}\right| \leqslant \cdot \varepsilon^{\alpha}\right)$, 使得对位于 $B\left(P_{\varepsilon}, \cdot \varepsilon^{\alpha}\right)\left(\alpha \geqslant \frac{N}{4}\right)$ 的任意 $P$, 成立

$$
\sup _{x}\left|u^{\varepsilon}(x, P)-\tilde{u}_{N}^{\varepsilon}(x, P)\right| \leqslant \cdot \varepsilon^{M},
$$

这里 $u^{\varepsilon}(x, P)$ 满足方程 $H_{\varepsilon}\left(P+D_{x} u^{\varepsilon}, x\right)=\bar{H}_{\varepsilon}(P), \tilde{u}_{N}^{\varepsilon}$ 如 $(2.3)$ 式所示.

证明 定义新坐标 $(P, X)$ 如下:

$$
\left\{\begin{array}{c}
p=P+D_{x} \tilde{u}_{N}^{\varepsilon}(x, P), \\
X=x+D_{P} \tilde{u}_{N}^{\varepsilon}(x, P),
\end{array}\right.
$$

定义 $(x(0), p(0))=\left(x_{0}, P_{1}+D_{x} u^{\varepsilon}\left(x_{0}, P_{1}\right)\right)$, 这里 $x_{0}$ 依赖于 $P_{1}, P_{1}$ 属于 $B\left(P_{\varepsilon}, \cdot \varepsilon^{\alpha}\right)$ 并且 $x_{0}$ 和 $P_{1}$ 满足

$$
D_{x} u^{\varepsilon}\left(x_{0}, P_{1}\right)=D_{x} \tilde{u}_{N}^{\varepsilon}\left(x_{0}, P_{1}\right) .
$$

$(x(0), p(0))$ 是 Hamilton 方程

$$
\left\{\begin{array}{l}
\dot{x}=-D_{p} H_{\varepsilon}(p, x), \\
\dot{p}=D_{x} H_{\varepsilon}(p, x),
\end{array}\right.
$$


相轨线 $(x(t), p(t))$ 的初值点. 易知 $P(0)=P_{1}$. 在新坐标 $(X, P)$ 下考察 Hamilton 方程, 初值 条件记为 $(X(0), P(0))$, 则

$$
\left\{\begin{array}{l}
\dot{X}=-D_{P} \tilde{H}_{\varepsilon}(P)+O\left(\varepsilon^{N}+\left|P-P_{0}\right|^{N-1}\right), \\
\dot{P}=O\left(\varepsilon^{N}+\left|P-P_{0}\right|^{N}\right) .
\end{array}\right.
$$

同文献 [1], 我们有下面的性质:

性质 2.1

$$
\sup _{0 \leqslant t \leqslant \frac{1}{\varepsilon^{(N-1) / 2}}}\left|P(t)-P_{1}\right| \leqslant \cdot \varepsilon^{\frac{N}{4}}
$$

这里

$$
5<N \leqslant-1-\frac{1}{2} \log _{2}^{\varepsilon} .
$$

证明 如同文献 [1], 我们有

$$
\frac{d}{d t}\left|P(t)-P_{1}\right|^{2} \leqslant 2^{N+1}\left|P(t)-P_{1}\right| \varepsilon^{N}+2^{N+1}\left|P(t)-P_{1}\right|^{N+1} .
$$

若 $\left|P(t)-P_{1}\right|^{N-1} \leqslant \varepsilon^{N}$, 则

$$
(2.9) \text { 式 } \leqslant 2^{N+1} \varepsilon^{N}+2^{N+1} \varepsilon^{N}\left|P(t)-P_{1}\right|^{2} \text {. }
$$

由 $P(0)=P_{1}$ 及 Gronwall 不等式, 有

$$
\left|P(T)-P_{1}\right|^{2} \leqslant e^{2^{N+1} \varepsilon^{N} T} 2^{N+1} \varepsilon^{N} T .
$$

若选 $N \leqslant-1-\frac{1}{2} \log _{2}^{\varepsilon}, T=\frac{1}{\varepsilon^{(N-1) / 2}}$, 将有

$$
\left|P(T)-P_{1}\right|^{2} \leqslant \cdot \varepsilon^{\frac{N}{2}} .
$$

注意 $N>5$, 结论是明显的.

注 2.3 从性质 2.1, 知道若 $\varepsilon$ 固定, 则 $N$ 有个自然的限制. 这也是我们下面为何如第 3 节那样选择 $\alpha, N$ 和 $M$ 的原因所在.

为简便起见, 我们用 $\phi\left(x, P_{1}\right)$ 来记 $X=x+D_{P} \tilde{u}_{N}^{\varepsilon}\left(x, P_{1}\right)$. 注意到当 $\varepsilon$ 充分小时, $\phi_{\varepsilon}\left(x, P_{1}\right)=$ $X$ 是一微分同胚. 在下面, 我们将把记号 $\phi_{\varepsilon}\left(x, P_{1}\right)$ 中的 $P_{1}$ 省掉. 记

$$
U(X)=u^{\varepsilon}\left(\phi_{\varepsilon}^{-1}(X), P_{1}\right)-\tilde{u}_{N}^{\varepsilon}\left(\phi_{\varepsilon}^{-1}(X), P_{1}\right) .
$$

同文献 [1], 当 $0 \leqslant t \leqslant \frac{1}{\varepsilon^{(N-1) / 2}}$ 同时 $N$ 满足 $(2.8)$ 式时, 成立

$$
\frac{d}{d t} U(X(t))=\left(P(t)-P_{1}+O\left(\varepsilon^{\frac{N}{4}}\right)\right) D_{p} H_{\varepsilon}(p(t), x(t))=O\left(\varepsilon^{\frac{N}{4}}\right) .
$$

我们将给 $\tilde{u}_{N}^{\varepsilon}$ 加一个合适的常数使之满足 $U(X(0))=0$. 例如用 $\tilde{u}_{N}^{\varepsilon}+\left(u^{\varepsilon}\left(x_{0}, P_{1}\right)-\right.$ $\left.\tilde{u}_{N}^{\varepsilon}\left(x_{0}, P_{1}\right)\right)$ 取代 $(2.10)$ 式中的 $\tilde{u}_{N}^{\varepsilon}$. 这样沿着相轨线得到

$$
\sup _{0 \leqslant t \leqslant \frac{1}{\varepsilon^{N / 8}}} U(X(t))=O\left(\varepsilon^{\frac{N}{8}}\right) .
$$

由 (2.7) 式, 有 $\dot{X}=-D_{P} \tilde{H}_{\varepsilon}(P)+O\left(\varepsilon^{N}+\left|P-P_{0}\right|^{N-1}\right)$. 当 $0 \leqslant t \leqslant \frac{1}{\varepsilon^{(N-1) / 2}}$ 时, 明显成 立 $\left|P(t)-P_{1}\right| \leqslant O\left(\varepsilon^{\frac{N}{4}}\right)$. 故, 当 $0 \leqslant t \leqslant \frac{1}{\varepsilon^{(N-1) / 2}}$ 同时 $\alpha \geqslant \frac{N}{4}$ 时, 将有

$$
\begin{aligned}
D_{P} \tilde{H}_{\varepsilon}(P(t)) & =D_{P} \tilde{H}_{\varepsilon}\left(P_{1}+O\left(\varepsilon^{\frac{N}{4}}\right)\right) \\
& =D_{P} \tilde{H}_{\varepsilon}\left(P_{\varepsilon}+O\left(\varepsilon^{\alpha}\right)+O\left(\varepsilon^{\frac{N}{4}}\right)\right) \\
& =D_{P} \tilde{H}_{\varepsilon}\left(P_{\varepsilon}+O\left(\varepsilon^{\frac{N}{4}}\right)\right) \\
& =\omega_{0}+O\left(\varepsilon^{\frac{N}{4}}\right) .
\end{aligned}
$$


上面最后一个等式来自于引理 2.3 . 这样, 我们有

$$
\dot{X}=-\omega_{0}+O\left(\varepsilon^{\frac{N}{4}}\right) .
$$

由文献 [10](类似的方法见文献 [11,12]), 我们知道 Hamilton 相流及其 $\varepsilon^{M}$ 的宽度在 $T=$ $O\left(\frac{1}{\varepsilon^{M \tau}}\right)$ 内覆盖了整个环面. 若 $M \leqslant \frac{N}{8 \tau}$, 对于满足 $0 \leqslant t \leqslant \frac{1}{\varepsilon^{\frac{N}{8}}}$ 的某个时间, 有

$$
|X-X(t)| \leqslant O\left(\varepsilon^{M}\right) .
$$

这样, 由引理 4.1 及 4.2 , 有

$$
\begin{aligned}
|U(X)| & \leqslant|U(X)-U(X(t))|+|U(X(t))| \\
& \leqslant C|X-X(t)|+O\left(\varepsilon^{\frac{N}{8}}\right) \\
& \leqslant O\left(\varepsilon^{M}\right),
\end{aligned}
$$

这里 $P_{1} \in B\left(P_{\varepsilon}, \varepsilon^{\alpha}\right)\left(\alpha \geqslant \frac{N}{4}\right)$.

由注 2.1 和 2.2 及同文献 [1] 完全相同的方法, 有下面的性质 2.2 及定理 2.2 .

性质 2.2 假设 $\omega_{0}=D_{P} H_{0}\left(P_{0}\right)$ 是 Diophantine, $\tilde{u}_{N}^{\varepsilon}$ 同 (2.3) 式, 则

$$
\frac{1}{T} \int_{0}^{T} \frac{\gamma}{2}\left|D_{x} u^{\varepsilon}\left(x(t), P_{1}\right)-D_{x} \tilde{u}_{N}^{\varepsilon}\left(x(t), P_{1}\right)\right|^{2} d t \leqslant C_{1} \varepsilon^{N}+\frac{2}{T} \sup _{x}\left|u^{\varepsilon}-\tilde{u}_{N}^{\varepsilon}\right|,
$$

这里的积分沿着轨线 $x(t)$ 和 $x(\cdot)$ 满足方程

$$
\dot{x}(t)=-D_{p} H_{\varepsilon}\left(P_{1}+D_{x} u^{\varepsilon}\left(x(t), P_{1}\right), x(t)\right) .
$$

上面的常数 $C_{1}$ 不依赖于 $P_{1}, P_{1} \in B\left(P_{\varepsilon}, \cdot \varepsilon^{\alpha}\right)$.

定理 2.2 任给常数 $M>0$. 假设 $\omega_{0}=D_{p} H_{0}\left(P_{0}\right)$ 是 Diophantine, 使得 cell 方程 (1.1) 存在一个近似解 $\tilde{u}_{N}^{\varepsilon}$, 这里 $N$ 及 $\alpha$ 同定理 1 , 则

$$
\underset{x}{\operatorname{esssup}}\left|D_{x} u^{\varepsilon}\left(x, P_{1}\right)-D_{x} \tilde{u}_{N}^{\varepsilon}\left(x, P_{1}\right)\right| \leqslant C_{2} \varepsilon^{\frac{M}{2}},
$$

这里 $C_{2}$ 不依赖于 $P_{1}, P_{1} \in B\left(P_{\varepsilon}, \cdot \varepsilon^{\alpha}\right)$.

\section{3 弱的正则性}

本节将运用前面的定理 1 和 2 得到有关粘性解弱的正则性的定理. 为此我们把 $P$ 限制 在一个环域中, 具体而言就是 $P$ 满足 $\cdot \varepsilon^{2 \alpha} \leqslant\left|P-P_{\varepsilon}\right| \leqslant \cdot \varepsilon^{\alpha}$. 由定理 1 , 我们有

$$
\begin{aligned}
& \left|u^{\varepsilon}(x, P)-\tilde{u}_{N}^{\varepsilon}(x, P)-\left(u^{\varepsilon}\left(x_{0}, P\right)-\tilde{u}_{N}^{\varepsilon}\left(x_{0}, P\right)\right)\right| \leqslant \cdot \varepsilon^{M} \leqslant \cdot\left|P-P_{\varepsilon}\right|^{\frac{M}{2 \alpha}}, \\
& \left|u^{\varepsilon}\left(x, P_{\varepsilon}\right)-\tilde{u}_{N}^{\varepsilon}\left(x, P_{\varepsilon}\right)-\left(u^{\varepsilon}\left(x_{1}, P_{\varepsilon}\right)-\tilde{u}_{N}^{\varepsilon}\left(x_{1}, P_{\varepsilon}\right)\right)\right| \leqslant \cdot \varepsilon^{M} \leqslant \cdot\left|P-P_{\varepsilon}\right|^{\frac{M}{2 \alpha}} .
\end{aligned}
$$

进一步, 由引理 4.3 并注意到 $\alpha \geqslant \frac{N}{4}$ 及 $M \leqslant \frac{N}{8 \tau}$, 有

$$
\begin{aligned}
& \left|\left(u^{\varepsilon}(x, P)-u^{\varepsilon}\left(x, P_{\varepsilon}\right)\right)-\left(u^{\varepsilon}\left(x_{1}, P_{\varepsilon}\right)-\tilde{u}_{N}^{\varepsilon}\left(x_{1}, P_{\varepsilon}\right)\right)-\left(u^{\varepsilon}\left(x_{0}, P\right)-\tilde{u}_{N}^{\varepsilon}\left(x_{0}, P\right)\right)\right| \\
& \quad \leqslant\left|u^{\varepsilon}\left(x, P_{\varepsilon}\right)-\tilde{u}_{N}^{\varepsilon}\left(x, P_{\varepsilon}\right)-\left(u^{\varepsilon}\left(x_{1}, P_{\varepsilon}\right)-\tilde{u}_{N}^{\varepsilon}\left(x_{1}, P_{\varepsilon}\right)\right)\right| \\
& \quad+\left|u^{\varepsilon}(x, P)-\tilde{u}_{N}^{\varepsilon}(x, P)-\left(u^{\varepsilon}\left(x_{0}, P\right)-\tilde{u}_{N}^{\varepsilon}\left(x_{0}, P\right)\right)\right|+\left|\tilde{u}_{N}^{\varepsilon}(x, P)-\tilde{u}_{N}^{\varepsilon}\left(x, P_{\varepsilon}\right)\right| \\
& \quad \leqslant \cdot\left|P-P_{\varepsilon}\right|^{\frac{M}{2 \alpha}}+\cdot \varepsilon^{1+\alpha} \\
& \quad \leqslant \cdot\left|P-P_{\varepsilon}\right|^{\frac{M}{2 \alpha}}+\cdot\left|P-P_{\varepsilon}\right|^{\frac{1+\alpha}{2 \alpha}} \\
& \quad \leqslant \cdot\left|P-P_{\varepsilon}\right|^{\frac{M}{2 \alpha}} .
\end{aligned}
$$


定义

$$
\left\|u^{\varepsilon}(x, P)-u^{\varepsilon}\left(x, P_{\varepsilon}\right)\right\|=\inf _{c}\left|u^{\varepsilon}(x, P)-u^{\varepsilon}\left(x, P_{\varepsilon}\right)-c\right| .
$$

若选择 $M=\frac{N}{8 \tau}$ 并固定 $N$ 满足 (2.8) 式, 除此之外, 进一步选择 $\alpha=2^{k} N(k=0,1, \cdots)$, 则明显 的成立 $\left\|u^{\varepsilon}(x, P)-u^{\varepsilon}\left(x, P_{\varepsilon}\right)\right\| \leqslant \cdot\left|P-P_{\varepsilon}\right|^{\frac{1}{2^{k+4} \tau}}$. 对于 $\cdot \varepsilon^{2^{k+1} N} \leqslant\left|P-P_{\varepsilon}\right| \leqslant \cdot \varepsilon^{2^{k} N}(k=0,1, \cdots)$ 中的所有的 $P$ 而言, 有

$$
\left\|u^{\varepsilon}(x, P)-u^{\varepsilon}\left(x, P_{\varepsilon}\right)\right\| \leqslant \cdot\left|P-P_{\varepsilon}\right|^{\frac{1}{2^{k+4} \tau}} .
$$

在下面的定理中, 固定 $N=8$.

定理 3 对于满足 $\left|P-P_{\varepsilon}\right| \leqslant \cdot \varepsilon^{8}$ 的任意 $P$, 存在 $k_{0}$, 使得 $\cdot \varepsilon^{2^{k_{0}+4}} \leqslant\left|P-P_{\varepsilon}\right| \leqslant$ $\cdot \varepsilon^{2^{k_{0}+3}}\left(k_{0}=0,1, \ldots\right)$, 则对于环域中的 $P$ 而言,

$$
\left\|u^{\varepsilon}(x, P)-u^{\varepsilon}\left(x, P_{\varepsilon}\right)\right\| \leqslant \cdot P-\left.P_{\varepsilon}\right|^{\frac{1}{2^{k_{0}+4} \tau}} .
$$

由引理 4.4 和同上类似的办法, 我们有下面定理:

定理 4 对于满足 $\left|P-P_{\varepsilon}\right| \leqslant \cdot \varepsilon^{8}$ 的任意 $P$, 存在 $k_{0}$, 使得 $\cdot \varepsilon^{2^{k_{0}+4}} \leqslant\left|P-P_{\varepsilon}\right| \leqslant$ $\cdot \varepsilon^{2^{k_{0}+3}}\left(k_{0}=0,1, \ldots\right)$, 则对于环域中的 $P$ 而言,

$$
\underset{x}{\operatorname{esssup}}\left|D_{x} u^{\varepsilon}(x, P)-D_{x} u^{\varepsilon}\left(x, P_{\varepsilon}\right)\right| \leqslant \cdot\left|P-P_{\varepsilon}\right|^{\frac{1}{2^{k_{0}+5} \tau}} .
$$

\section{附录 A}

引理 2.3 的证明 由基本的计算, 我们有

$$
\begin{aligned}
\omega-\omega_{0}= & \frac{\partial^{2} \bar{H}_{0}}{\partial P^{2}}\left(P_{0}\right)\left(P-P_{\varepsilon}\right) \\
& +\left\{\frac{1}{2 !} \frac{\partial^{3} \bar{H}_{0}}{\partial P^{3}}\left(P_{0}\right)\left[\left(P-P_{0}\right)^{2}-\left(P_{\varepsilon}-P_{0}\right)^{2}\right]+\frac{\varepsilon}{1 !} \frac{\partial^{2} \bar{H}_{1}}{\partial P^{2}}\left(P_{0}\right)\left(P-P_{\varepsilon}\right)\right\}+\cdots+\Delta,
\end{aligned}
$$

这里

$$
\begin{aligned}
\Delta= & \frac{1}{(N-2) !} \frac{\partial^{N-1} \bar{H}_{0}}{\partial P^{N-1}}\left(P_{0}\right)\left[\left(P-P_{0}\right)^{N-2}-\left(P_{\varepsilon}-P_{0}\right)^{N-2}\right] \\
& +\cdots+\frac{\varepsilon^{N-3}}{1 !} \frac{\partial^{2} \bar{H}_{N-3}}{\partial P^{2}}\left(P_{0}\right)\left(P-P_{\varepsilon}\right) .
\end{aligned}
$$

直接估计可知

$$
\left|\frac{1}{(N-2) !} \frac{\partial^{N-1} \bar{H}_{0}}{\partial P^{N-1}}\left(P_{0}\right)\left[\left(P-P_{0}\right)^{N-2}-\left(P_{\varepsilon}-P_{0}\right)^{N-2}\right]\right| \leqslant \frac{\left(2^{N-2}-1\right)}{(N-2) !} \varepsilon^{N+\alpha-3} .
$$

类似地, 有

$$
|\Delta| \leqslant \cdot \sum_{t=1}^{N-2} \frac{2^{t}-1}{t !} \varepsilon^{\alpha+N-3} \leqslant \cdot \varepsilon^{\alpha+N-3} .
$$

故

$$
\left|\omega-\omega_{0}\right| \leqslant \cdot \varepsilon^{\alpha}+\cdot \varepsilon^{\alpha+1}+\cdots+\cdot \varepsilon^{N+\alpha-3} \leqslant \cdot \varepsilon^{\alpha} .
$$

下面的引理是明显的:

引理 A.1 $P_{1}$ 属于一紧集, 则 


$$
|x-y|=\left|\phi_{\varepsilon}^{-1}(X)-\phi_{\varepsilon}^{-1}(Y)\right| \leqslant \cdot|X-Y| .
$$

引理 A.2 $D$ 是 $\mathbb{R}^{n}$ 中一紧集, 则

$$
\left|u^{\varepsilon}(x, P)-u^{\varepsilon}(y, P)\right| \leqslant K|x-y|,
$$

这里 $P \in D$, 常数 $K$ 仅依赖于 $D$.

证明 记 $W_{P}$ 为 $x \in \mathbb{T}^{n}$ 中所有使得 $D_{x} u^{\varepsilon}(x, P)$ 存在的点的集合. 既然 $H_{\varepsilon}(p, x)$ 对 $p$ 凸, 我们知集合 $\left\{y: H_{\varepsilon}(y, x)=\bar{H}(P), P \in D\right\}$ 是 $\mathbb{R}^{n}$ 中一紧集, 故存在一正数 $K$, 使得对于任意 的 $p \in D, x \in W_{P}$, 有 $\left\|D_{x} u^{\varepsilon}(x, P)\right\| \leqslant K$ 成立.

对于任意的 $P \in D, u^{\varepsilon}(x, P)$ 是 Lipschitz 函数, 从而 $\mathbb{T}^{n} \backslash W_{P}$ 是零测集.

给定环面上的两点 $x$ 和 $y$, 由 Fubini 定理, 存在两子列 $x_{n}$ 和 $y_{n}$, 使得 $x_{n} \rightarrow x, y_{n} \rightarrow y$, 并且其两点的连线 $\Gamma_{n}: \gamma_{n}(t)=x_{n}+\frac{y_{n}-x_{n}}{\left|y_{n}-x_{n}\right|} t$ 交 $W_{P}$ 在 $\Gamma_{n}$ 上是全测度集, 从而有

$$
\begin{aligned}
\left|u^{\varepsilon}\left(y_{n}, P\right)-u^{\varepsilon}\left(x_{n}, P\right)\right| & \leqslant \int_{0}^{\left\|y_{n}-x_{n}\right\|}\left|\left\langle D_{x} u^{\varepsilon}(x, P), \dot{\gamma}_{n}\right\rangle\right| d t \\
& \leqslant \int_{0}^{\left\|y_{n}-x_{n}\right\|}\left\|D_{x} u^{\varepsilon}(x, P)\right\|\left\|\dot{\gamma}_{n}\right\| d t \\
& \leqslant K\left\|y_{n}-x_{n}\right\| .
\end{aligned}
$$

既然 $u^{\varepsilon}(x, P)$ 连续, $x_{n} \rightarrow x, y_{n} \rightarrow y$, 求极限可知

$$
\left|u^{\varepsilon}(y, P)-u^{\varepsilon}(x, P)\right| \leqslant K|y-x| \text {. }
$$

引理 A.3

$$
\left|\tilde{u}_{N}^{\varepsilon}(x, P)-\tilde{u}_{N}^{\varepsilon}\left(x, P_{\varepsilon}\right)\right| \leqslant \cdot \varepsilon^{1+\alpha} .
$$

\section{证明 直接估计知}

$$
\left|\frac{\partial^{N-t-1} v_{t}}{\partial P^{N-t-1}}\left(x, P_{0}\right)\left(P-P_{0}\right)^{N-t-1}-\frac{\partial^{N-t-1} v_{t}}{\partial P^{N-t-1}}\left(x, P_{0}\right)\left(P_{\varepsilon}-P_{0}\right)^{N-t-1}\right| \leqslant \cdot \varepsilon^{\alpha+N-t-2},
$$

这里 $t=1, \ldots, N-1$. 以下的推理是明显的.

类似地我们有

引理 A.4

$$
\left|D_{x} \tilde{u}_{N}^{\varepsilon}(x, P)-D_{x} \tilde{u}_{N}^{\varepsilon}\left(x, P_{\varepsilon}\right)\right| \leqslant \cdot \varepsilon^{1+\alpha} .
$$

\section{参考文献}

1 Gomes D. Perturbation theory for viscosity solutions of Hamilton-Jacobi equations and stability of AubryMather sets. SIAM J Math Anal, 35: 135-147 (2003)

2 Mather J. Modulus of continuity for Peierls's barrier. In: Rabinowitz P H, et al. eds. Periodic solutions of Hamiltonian Systems and related topics, 1987, 177-202

3 Bardi M, Capuzzo-Dolcetta I. Optimal control and viscosity solutions of Hamilton-Jacobi-Bellman equations. Boston: Birkhäuser Inc, 1997

4 Fathi A. Weak KAM theorem in Lagrangian dynamics. Cambridge: Cambridge University Press, 2007

5 Bessi U. Aubry-Mather theory and Hamilton-Jacobi equations. Comm Math Phys, 235: 495-511 (2003) 
6 E W. Aubry-Mather theory and periodic solutions of the forced Burgers equation. Comm Pure Appl Math, 52: $811-828$ (1999)

7 Fathi A, Siconolfi A. Existence of $C^{1}$ critical subsolutions of the Hamilton-Jacobi equation. Invent Math, 155: 363-388 (2004)

8 Gomes D. Regularity theory for Hamilton-Jacobi equations. J Differential Equations, 187: 359-374 (2003)

9 Lions P L, Papanicolao G, Varadhan S R S. Homogeneization of Hamilton-Jacobi equations. Preliminary Version, 1988

10 Bourgain J, Golse F, Wennberg B. On the distribution of free path lengths for the periodic Lorentz gas. Comm Math Phys, 190: 491-508 (1998)

11 Dumas H S, Ergodization rates for linear flow on the torus. J Differential Equations, 3: 593-610 (1991)

12 Dumas H S, Dumas L, Golse F. On the mean free path for a periodic array of spherical obstacles. J Statist Phys, 82: 1385-1407 (1996) 